\title{
Comentario Editorial: Tratamiento de la tuberculosis: niveles, barreras y facilitadores
}

\author{
Tuberculosis treatment: levels, barriers and facilitators
}

A pesar de un mayor esfuerzo global en el control y la disponibilidad de medicamentos eficaces de bajo costo, la tuberculosis sigue siendo un desafío en muchos lugares del mundo y pone de manifiesto algunas debilidades del sistema de salud. Desde hace años, la Organización Mundial de la Salud recomienda la estrategia de tratamiento supervisado (TDO) aunque en la Argentina no ha sido ampliamente implementada en algunas provincias ${ }^{1}$. En nuestro país, apenas el 54 por ciento de los casos con baciloscopía positiva tienen TDO documentado y en la provincia de Buenos Aires (donde se concentra el 50 por ciento de carga de la enfermedad del país) representan menos del 30 por ciento, aún cuando las tasas reportadas de tratamiento exitoso con TDO están cercanas a la meta del 85 por ciento, mientras que las del auto-administrado se mantienen por debajo del 75 por ciento. Por otro lado, el grado de supervisión directa del tratamiento es muy variable, tanto entre países como fronteras adentro de un mismo país ${ }^{1,2}$.

El uso de diferentes modalidades y los posibles factores asociados a las aún insuficientes tasas de éxito del tratamiento no han sido sistemáticamente evaluadas ${ }^{3}$.

Un gran número de estudios cualitativos se ha enfocado en el tema de la adherencia de los pacientes al tratamiento antituberculoso ${ }^{4}$. La adherencia a una conducta gira en torno a una multitud de factores complejos y dinámicos entre factores individuales, sociales, estructurales y del sistema que interactúan para determinar diferentes respuestas.

En el caso de la salud en general y de la tuberculosis en particular, la mayoría de los pacientes no tienen una libre elección, sino que sus respuestas están fuertemente condicionadas por el contexto en el que están inmersos. El concepto de la adherencia a los tratamientos es de por sí multifacético y la responsabilidad debe recaer tanto en los individuos como en el sistema de salud. No es prudente ni aconsejable juzgar los resultados de un programa si no se revisan los procesos que conducen a dichos resultados.

En el Instituto de Efectividad Clínica y Sanitaria (IECS) realizamos un estudio cuyo objetivo principal fue identificar las estrategias de tratamiento y explorar las barreras y facilitadores relacionados con el éxito del tratamiento, desde la perspectiva de los pacientes, familiares, personal sanitario, y responsables locales, regionales y nacionales del programa de tuberculosis ${ }^{5}$. Algunos de los hallazgos interesantes se refieren a las diferentes estrategias de tratamiento que se utilizan, a las intervenciones creativas adaptadas por los profesionales en los centros de salud y a la importancia de fortalecer el compromiso, la continuidad y la colaboración.

Algunas de las recomendaciones que surgen de esta investigación proponen una mayor descentralización y extensión de las horas de atención, la implementación de incentivos o sub- sidios directos a los pacientes, evitar la rotación del personal de salud y la multitarea, la elección del supervisor del tratamiento y un sistema de información centralizado.

Aunque claramente influenciada por factores personales, sociales y estructurales, la elección final de la estrategia de tratamiento está estrechamente relacionada con el sistema y la organización de la atención. Recientemente, se ha enfatizado en la necesidad de una aplicación más "flexible" de la estrategia TDO y enfoques "centrados en el paciente" para adaptarse a las situaciones locales, recursos y medio ambiente $e^{6,78}$. Nuestro estudio identificó varios ejemplos de esta flexibilidad, como por ejemplo TDO negociado con la familia, extensión de horarios y, en algunos casos, la entrega de subsidios directos a los pacientes.

Los profesionales de la salud y los responsables políticos deberían tener en cuenta los factores que mantienen la existencia de la tuberculosis en las distintas comunidades, las barreras y facilitadores que influyen en el éxito del tratamiento y las estrategias exitosas que se implementan en forma aisla$\mathrm{da}^{8,9}$. Los factores individuales y sociales relacionados con el fracaso del tratamiento son muy similares en todos los estudios realizados en diferentes partes del mundo ${ }^{4}$, con diferentes contextos socioeconómicos y culturales, y son muy difíciles de cambiar, a menos que exista un fuerte compromiso político. Por otro lado, los factores relacionados con la organización del sistema y la prestación de los servicios de salud deben transformarse, ya que es en el mejor interés de la salud pública adaptarlos específicamente a los pacientes y su contexto, en lugar de intentar adaptar a los pacientes a los modelos en existencia. La "institucionalización" de los programas gubernamentales requiere visibilidad y el reconocimiento de las comunidades, un marco normativo para orientar las acciones comunes, un sistema confiable y eficiente de seguimiento y un presupuesto asignado para apoyar los recursos necesarios. Para lograr el éxito en el tratamiento de la tuberculosis se debe trabajar en el compromiso político, en una financiación sostenida, en la estandarización del tratamiento, en el soporte a los pacientes, en la implementación de un sistema que permita su seguimiento y en el fortalecimiento y la evaluación de impacto de los programas.

La realización de estudios de intervención sobre las barreras personales, sociales y del sistema resulta esencial para entender las causas de las bajas tasas de éxito del tratamiento.

No se pueden modificar fácilmente los determinantes estructurales de la salud, pero a la luz de los resultados de este y de otros estudios similares, podemos identificar factores del sistema que nos permitan superar algunas de las limitaciones actuales en el corto plazo, así como planificar un conjunto más ambicioso de medidas para mejorar los resultados en el largo plazo.

Rubinstein E. Tratamiento de la tuberculosis: niveles, barreras y facilitadores. Evid Act Pract Ambul. Abr-Jun 2012;15(2):45.

Referencias

1. World Health Organization. (2011). TB country profile Argentina, 2009. Disponible en URL: http://www.who.int/GlobalAtlas/predefinedReports/TB/PDF_Files/arg.pdf

2. Instituto Nacional de Enfermedades Respiratorias E. Coni. (2010). Resultado del tratamiento de los casos de tuberculosis. Situación nacional y por jurisdicción, República Argentina, 2007.

3. Lienhardt C. y col. Factors determining the outcome of treatment of adult smear-positive tuberculosis cases in The Gambia. Int J Tuberc Lung Dis 1998;2(9):712-718.

4. Munro, S. y col. Patient adherence to tuberculosis treatment: A systematic review of qualitative research. PLoS Med 2007:4(7), e238.

5. Iribarren, S. Rubinstein,F, Discacciati, V. Pearce,P. Barreras y Facilitadores para el Tratamiento exitoso de la Tuberculosis en áreas de alta carga en Provincia de Buenos Aires,

5. Iribarren, S. Rubinstein, $F$, Discac

Argentina: un Estudio Cualitativo.

6. Tuberculosis Coalition for Technical Assistance. International Standards for Tuberculosis Care (ISTC). Diagnosis, Treatment, Public Health. 2009, 2nd ed.

7. Macq JC. y col. An exploration of the concept of directly observed treatment (DOT) for tuberculosis patients: From a uniform to a customised approach. Int J Tuberc Lung Dis 2003;7(2):103-109.

8. Volmink J. y col. Directly observed therapy and treatment adherence. Lancet 2000;355(9212):1345-1350.

9. Pope DS., Chaisson, RE. TB treatment: As simple as DOT? Int J Tuberc Lung Dis. 2003;7(7):611-615. 